\title{
SUCCESSFUL TREATMENTS OF TWO HUMAN AVIAN INFLUENZA CHINESE CHILDREN
} AND FOLLOW-UP

\author{
Y.M. Zhu ${ }^{1}$, R.P. Luo ${ }^{2}$, Z.Y. Xu ${ }^{1}$, X.P. Zhang ${ }^{1}$, L. Tang ${ }^{1}$, J. Dong ${ }^{1}$ \\ ${ }^{1}$ Pediatric Intensive Care Unit, Children's Hospital of Hunan Province, ${ }^{2}$ Department of Infection, Children's \\ Hospital of Hunan Province, Changsha, China
}

Background and aims: Human H5N1 avian influenza caused worldwide attention in 1997 when the first case was found in Hong Kong, our present study is to summarize the clinical characteristics, diagnostic and therapeutic experiences of the first human case and the youngest case with human H5N1 avian influenza pneumonia Chinese children in mainland of China and further provide effective strategies for the prevention and cure of future such children.

Methods: To analyze the clinical data of two pneumonia children and following up data.

Results: On October 18, 2005, a 9-year-old boy, fever, dry cough, on day 7 after onset, his body temperature reached $40^{\circ} \mathrm{C}$, large areas of consolidation on chest X-ray, decreased peripheral blood leukocyte counts and most neutrophilic granulocytes. After therapies with amantadine and glucocorticoid, he was the first human case in mainland of China and successfully treated and discharged. On January 9, 2006, another 6-year-old boy, high fever, cough, polypnea, the left lung consolidation on chest X-ray, less hemogram. After therapies with short-term symmetrel and ribavirin, low-dosage glucocorticoid for 4 weeks and CPAP assistant ventilations, he also was the youngest avian influenza case in China and successfully treated and discharged. At present, two boys are very healthy.

Conclusions: It is very important to pay much more attention on epidemiological contact history and clinical characteristics of avian influenza patients, earlier detect and diagnose and therapy as soon as possible. These two cases children treated successfully attributed to timely discovery, promptly diagnosis and effectively treatments. 